\title{
The developing kidney actively negotiates geometric packing conflicts to avoid defects
}

\author{
Authors: Louis S. Prahl ${ }^{1}$, John M. Viola ${ }^{1}$, Jiageng Liu' ${ }^{1}$, Alex J. Hughes ${ }^{1,2,3,{ }^{*}}$
}

\begin{abstract}
Affiliations:
${ }^{1}$ Department of Bioengineering, University of Pennsylvania; Philadelphia, 19104, PA, USA.

${ }^{2}$ Department of Cell \& Developmental Biology, University of Pennsylvania; Philadelphia, 19104, PA, USA.

${ }^{3}$ Institute for Regenerative Medicine, University of Pennsylvania; Philadelphia, 19104, PA, USA.

${ }^{*}$ Corresponding author. Email: ajhughes@seas.upenn.edu
\end{abstract}

\begin{abstract}
:
The physiological functions of several organs rely on branched tubular networks, but little is known about conflicts in development between building enough tubules for adequate function and geometric constraints imposed by organ size. We show that the mouse embryonic kidney epithelium negotiates a physical packing conflict between tubule tip duplication and limited area at the organ surface. Imaging, computational, and soft material modeling of tubule 'families' identifies six geometric packing phases, including two defective ones. Experiments in kidney explants show that a retrograde tension on tubule families is necessary and sufficient for them to avoid defects by switching to a vertical orientation that increases packing density. These results reveal developmental contingencies in response to physical limitations, and create a framework for classifying kidney defects.
\end{abstract}

One-Sentence Summary: Epithelial branching in the kidney causes a geometric packing conflict that is resolved through internally generated tensions

\section{Main Text:}

Epithelial organs perform diverse functions - lung alveoli exchange gases, kidney tubules exchange water and ions, and mammary acini secrete milk. However, little is known about how developmental constraints and compromises affect adult organ function per unit volume (1-3). In the embryonic mouse kidney, the branching $\mathrm{ECAD}^{+} /$calbindin $^{+}$ureteric epithelium creates an arborized network of epithelial tubules linked by junctions or 'nodes' (Fig. 1A,B) through reciprocal signaling interactions with $\mathrm{SIX}^{+}$cap mesenchyme and PDGFRA ${ }^{+}$stroma (4). Ureteric tubule tips induce nephron formation (5) (Fig. 1B), so the eventual filtration and reabsorption function of the adult kidney likely scales with the number of tips. However, while some organs like the lung fill 3D space during branching, ureteric branching occurs at the kidney surface (6-8). This should create a conflict between the available organ surface area and exponentially increasing tip number (Supplementary Text). How then does the kidney navigate this conflict?

To investigate tip patterning as branching progresses, we examined whole mount mouse kidneys at developmental stages from embryonic day (E)14-E18 by confocal immunofluorescence (Fig. 1C). Each ureteric tip is surrounded by a dynamic swarm of cap mesenchyme cells within distinct domains near the surface (9). We quantified the tip domain area as well as the $\mathrm{SIX} 2^{+}$cap area and circularity at each stage (Fig. 1D). Tip domain area and cap area both steadily decreased with organ stage, converging after E16. This indicates closer 
bioRxiv preprint doi: https://doi.org/10.1101/2021.11.29.470441; this version posted December 1, 2021. The copyright holder for this preprint (which was not certified by peer review) is the author/funder, who has granted bioRxiv a license to display the preprint in perpetuity. It is made available under aCC-BY-NC-ND 4.0 International license.

packing of cap mesenchyme populations due to a reduction in residual area occupied by stroma and parent nodes (junctions between two daughter tips), which appeared to drop into the kidney volume more quickly after E15.5. Cap circularity also steadily decreased since caps conformed to the area available to them as neighboring ones encroached. This indicates a compromise between mutual tip repulsion and confinement caused by crowding of neighboring tips over time (Supplementary Text).

A

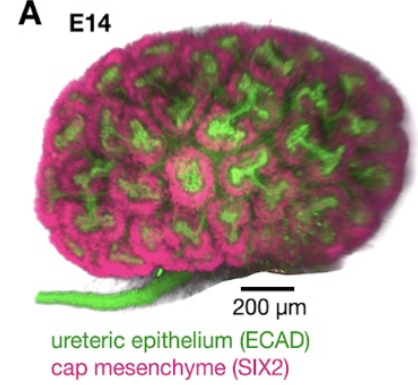

C

$C_{\text {E14 }}$ top $(x y)$

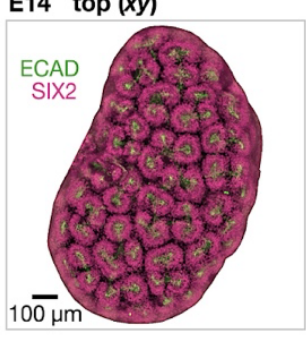

D

tip domain a

$50 \mathrm{~mm}$

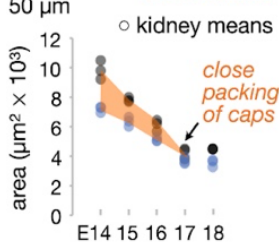

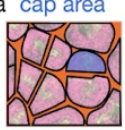

$5 \overline{\mu \mathrm{m}}$
E16

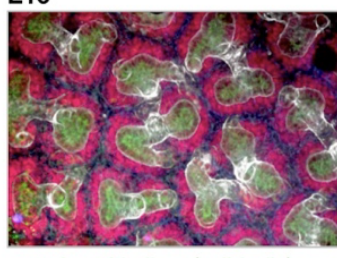

ureteric epithelium (calbindin) basement membrane (PNA) cap mesenchyme (SIX2) stroma (PDGFRa)
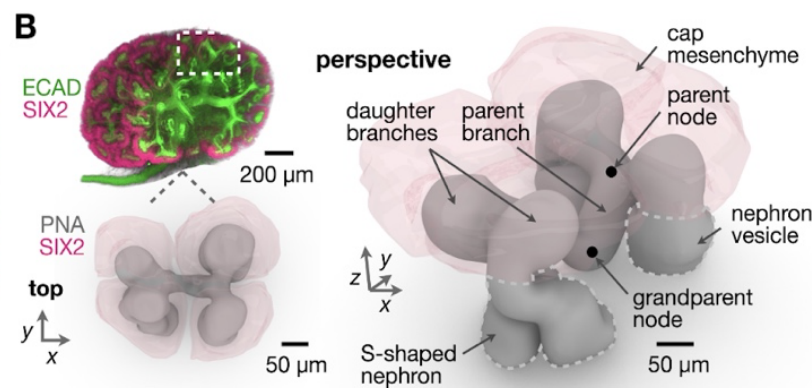

E16
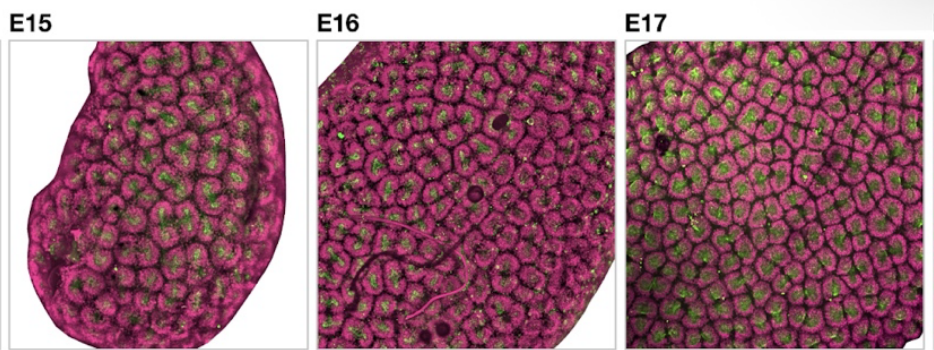

E18

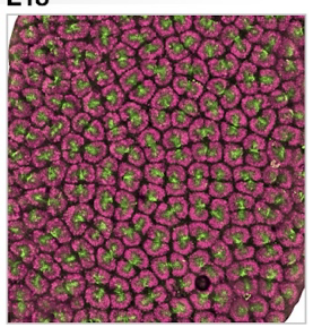

E

E15

E16
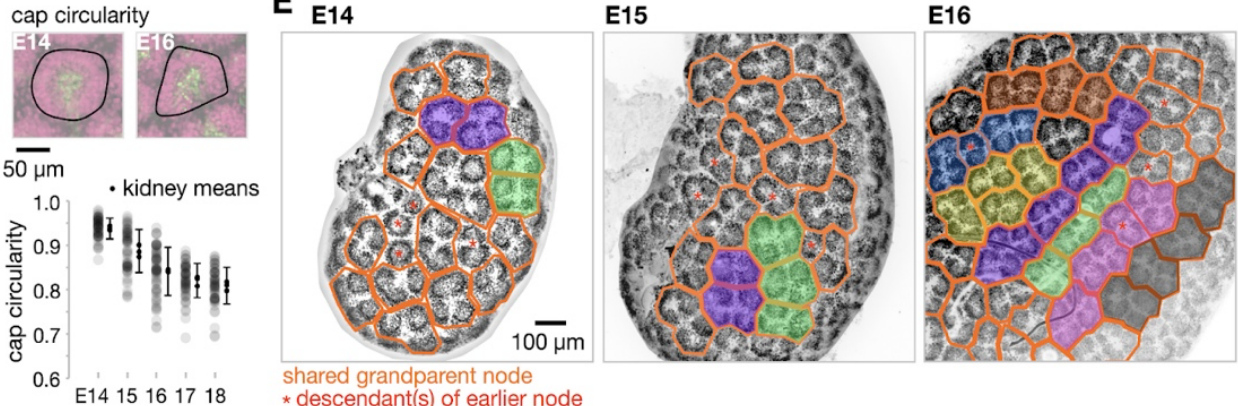

* descendant(s) of earlier node

Fig. 1. Tips of the ureteric epithelial tree form diverse patterns, pack closely, and achieve longrange order. (A) Left, 10x confocal projection of cleared mouse kidney at embryonic day (E)14. Right, detail of ureteric tips at E16. (B) Reconstruction of tubule family sharing a grandparent node. (C) 10x projections of kidneys at fixed zoom. (D) Quantitation of: left, tip domain (total area per tip) and cap mesenchyme area (means of $>12$ measurements per $n=3$ kidneys per time point); right, cap mesenchyme circularity ( $>18$ measurements per $n=3$ kidneys per time point). Error bars are \pm S.D. for all measurements per embryonic day. (E) Outlines of tubule families sharing a grandparent node. Fills indicate regions of aligned families. 
In similar physical problems, repulsive objects can adopt distinctive packing geometries or 'phases', with critical transitions between them depending on the amount of confinement (1012). We observed tubule families sharing a grandparent node progressively pack into aligned regions along the surface on the scale of 3-5 families at around E15-E16 (Fig. 1E, Fig. S1). We also observed dislocations and disclinations between aligned regions, which are hallmarks of liquid crystals and elastic crystalline solids $(13,14)$. These data and published accounts of a lack of stereotypy $(7,15)$ argue against a strict morphogenetic control program and instead for tip patterns being determined by competition between crowding and repulsion.

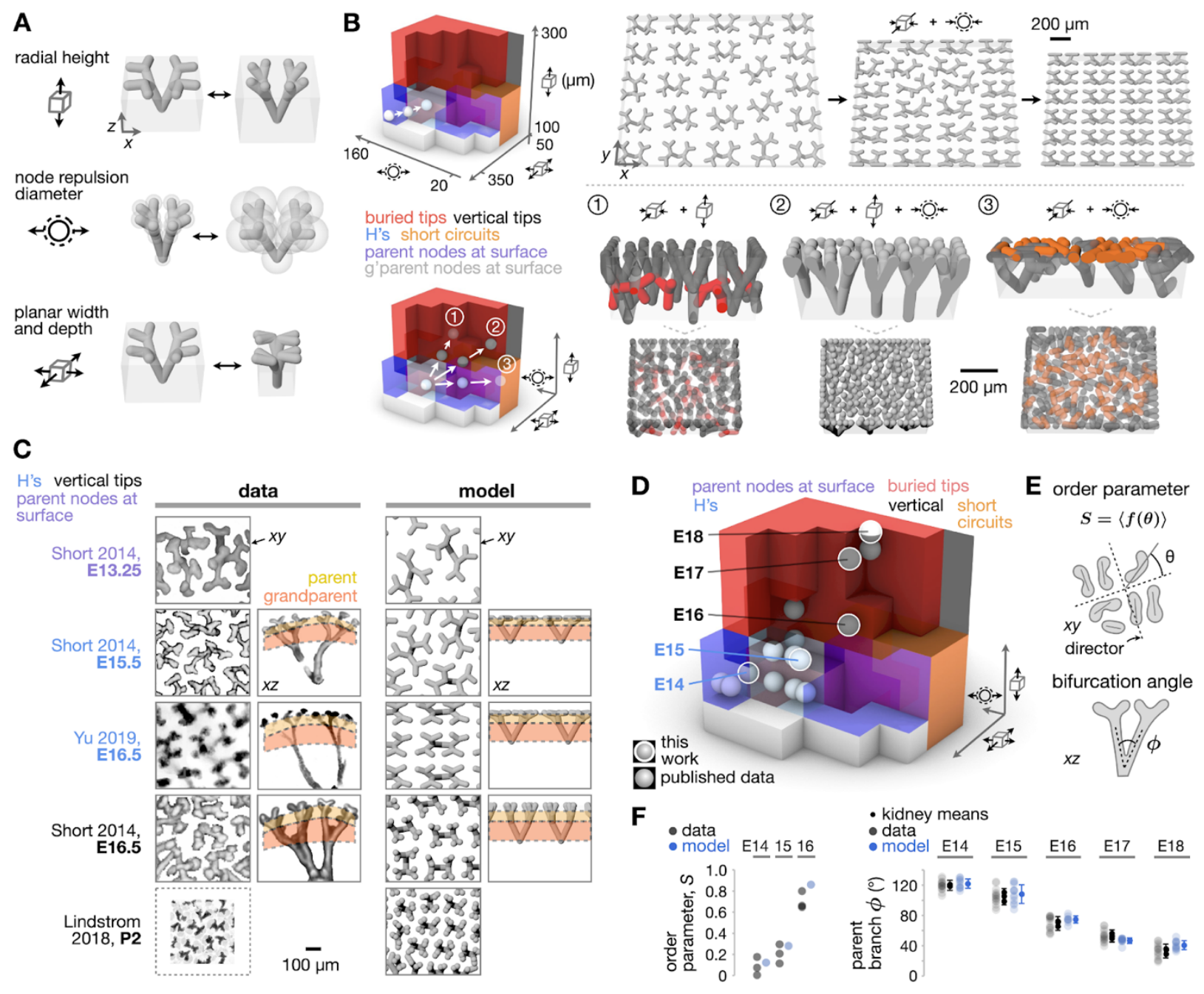

Fig. 2. Published and measured kidney data map to a trajectory through physics-based model space that avoids defects. (A) Model output demonstrating geometric parameters. (B) Left, 3D plot of six phases of tip patterns at the model surface. Top row, an example trajectory from low tip density in the parent-nodes-at-surface phase into the H's phase. Bottom row, three possible trajectories from the H's phase into the buried tips, vertical, and short circuits phases. Insets show side views of tip patterns where red segments are buried in the model volume, and orange segments intersect with other segments. (C) Left, Top and side views, where available, of published ureteric tubule tip patterns. Right, corresponding views from model cases with matching geometric parameters. (D) Published kidneys and kidneys measured in this work (means of $n=3$ kidneys per embryonic day) mapped to model phase space (see Fig. S3 for full detail). (E) Quantitative metrics. (F) Quantitation of metrics comparing kidneys from this work and model cases. Error bars at right are \pm S.D. for all measurements pooled across $n=3$ kidneys for each embryonic day. 
Can overcrowding of tips at the kidney surface cause geometric packing errors? If so, does the developing organ restructure tubule families to avoid these? To answer this, we made a computational model governed by three spatial parameters: 1) repulsion distance between tip centers, 2) planar confinement (width and depth) in the $x y$ plane of tubule families each sharing a great-grandparent node, and 3) radial height occupied by families in z (Fig. 2A, Movie S1, Supplementary Text). The model uses energy minimization to predict branch positions at steady state (Movie S2), rather than simulating branching itself. A parameter sweep revealed six phases of tubule packing with distinct morphologies, plus one trivial case where tubules are inefficiently packed (Fig. 2B, Fig. S2A). Three phases are defined by parent nodes sitting near the kidney surface - 'grandparent nodes at surface', 'parent nodes at surface', and 'H's'. The H's phase among these exhibits crystalline packing of families with long-range alignment. Two defective packing states appear as planar confinement increases. We call these states 'short circuits' where tubules intersect near the surface and 'buried tips' where surface overcrowding displaces some tips beneath the surface (Fig. 2B, Supplementary Text). Finally, a 'vertical packing' phase sees tips increasing their packing density by reorienting along the $z$ axis (Fig. S2B). Published examples of tip patterns qualitatively reflect increased orientational ordering of tips in $x y$ over $\sim E 13.25-E 16.5$, and a transition to vertical orientation after $\sim E 16$, in step with corresponding model cases (Fig. 2C).

We quantified this by mapping spatial parameters from our own and published images of tubule families to the model, finding that kidney development roughly progresses along a trajectory in phase space through the H's phase and ending with vertical packing at E17-E18 (Fig. 2D, Fig. S3) $(6,16,17)$. We then evaluated two metrics of tubule organization. First, an order parameter $S$ that measures local daughter branch alignment along orthogonal preferred directions (directors) when parent nodes are visible near the surface (Fig. 2E). $S=1$ indicates crystalline packing and perfect ordering of each daughter along either director (Supplementary Text). $S$ increased from E14-E16 as tubules entered the H's phase with close agreement between experiment and model (Fig. 2F). Second, we quantified the parent branch bifurcation angle $(\phi)$ (Fig. 2E) and found that this progressively decreased in both experiment and model between E14 and E18 as tips shifted toward vertical packing (Fig. 2F) $(16,18)$. The packing model thus explains developmental variation in tip patterns. It also predicts that a vertical packing switch is necessary for the developing kidney to increase tip density while avoiding defects.

We next asked whether physical forces could mediate the switch to vertical packing, since this requires that parent through great-grandparent nodes move further from the kidney surface (note increase in yellow and orange layer heights in Fig. 2C). Lindström et al. described 'node retraction' as retrograde movement of branch points towards the ureter beginning at $\sim \mathrm{E} 15$ (19), which is consistent with our data showing a reduction in $\phi$ during the shift to vertical packing (Fig. 2D,F). However, it is unclear whether this rearrangement is caused by revision of branch point locations through collective cell dynamics or by an active retraction force generated within the ureteric epithelium. We reasoned that a retrograde force on tubules $\left(F_{z}\right)$ could be indirectly measured by disrupting the basement membrane at the epithelial-mesenchymal interface using dispase (20). This would release adhesive and shear forces at that interface and cause tips to rapidly retract away from the kidney surface (Fig. 3A). Indeed, dispase added to E15 kidney explants caused basement membrane disruption, epithelial delamination from the surrounding mesenchyme and tip and parent node retraction away from the kidney surface in $<10$ mins (Fig. 3B,C, Movie S3). We found significant decreases in the tip areas at the surface relative to untreated controls that were sensitive to either the myosin II inhibitor blebbistatin or the ROCK inhibitor Y-27632 (Fig. 3C). In contrast, E14 kidneys showed less tubule retraction upon dispase treatment, but returned to the retraction level of E15 kidneys in the presence of calyculin $A$ (calA), which stimulates actomyosin contractility (21) (Fig. 3C). These data indicate that the 
epithelial-mesenchymal interface is subject to an actomyosin-based retraction force that switches on at $\sim$ E15 during the shift to vertical packing.

Is this retraction force then sufficient to shift tubule families from the crystalline H's state to more vertical packing? To test this, we added calA to intact E15 kidneys and cultured for $18 \mathrm{hr}$. We then measured $\phi$ and the radial distance of parent nodes from the kidney surface $\left(p_{z}\right)$ as metrics for the vertical transition of tip families (Fig. 3D). Indeed, $\phi$ decreased and $p_{z}$ increased, whether or not the ureter had been removed (Fig. 3E,F). This revealed that forces intrinsic to the ureteric tree are sufficient to explain the transition of tubule families to the vertical packing phase.

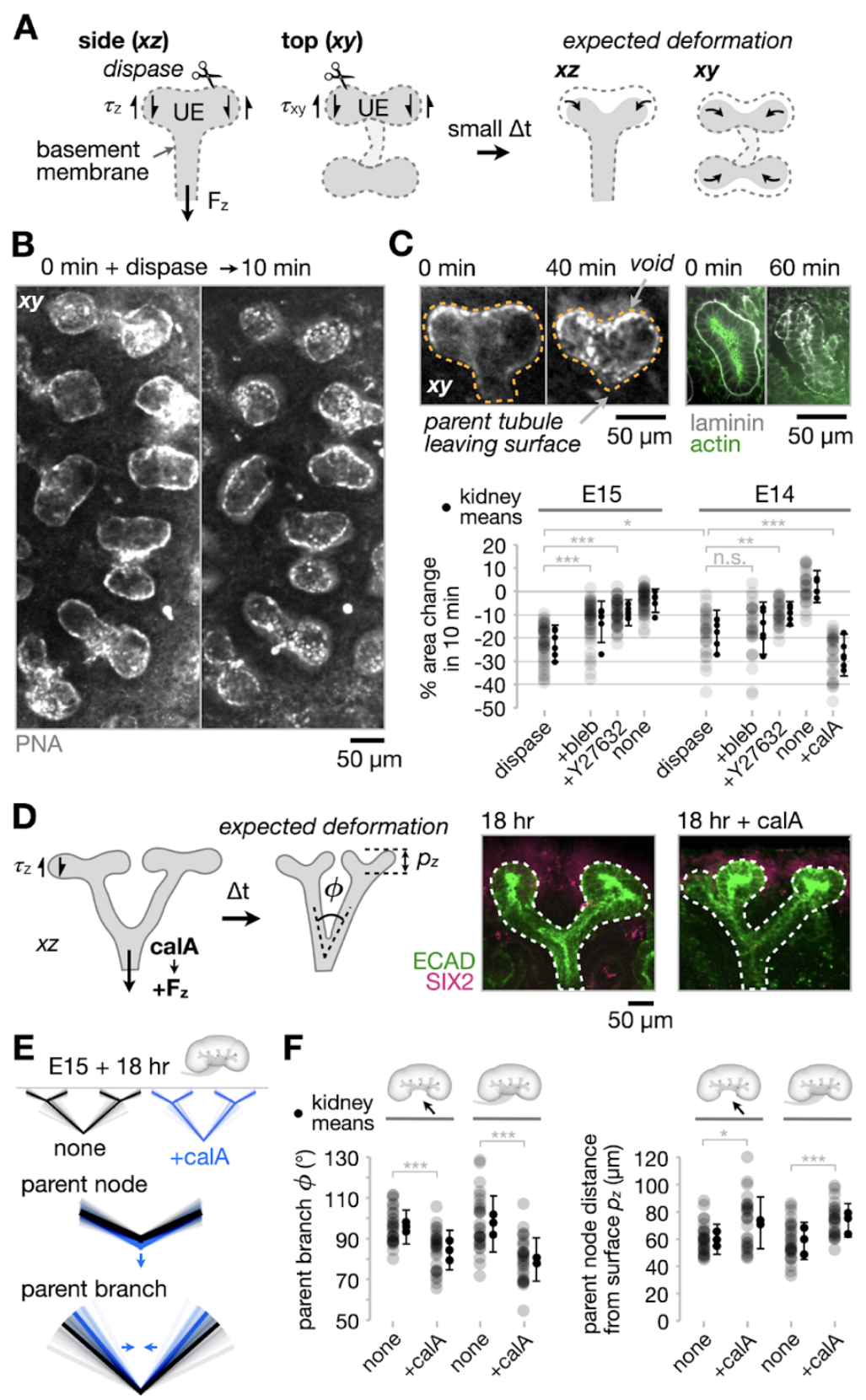

Fig. 3. Retrograde tubule tension is sufficient for transition to vertical packing. (A) Expected tip deformation after dispase. (B) Tip pattern in live E15 kidney explant before (left), and after $10 \mathrm{~min}$ dispase (right). (C) Top left, Example daughter tip pair. Top right, Laminin (basement membrane) immunofluorescence for similar pairs +/- dispase. Bottom, Tip area change after 10 min dispase; 60 min pre-treatment with $20 \mu \mathrm{M}$ blebbistatin (bleb), 20 $\mu \mathrm{M}$ Y-27632, or $25 \mathrm{nM}$ calyculin A (calA) followed by 10 min dispase; or no dispase (none) for E14 and E15 kidneys ( $>5$ measurements per $n=6$ kidneys per condition). Error bars are \pm S.D. across all measurements per condition. (D) Left, Expected tubule deformation with calA; $\phi=$ parent bifurcation angle, $p_{z}=$ parent node distance from kidney surface. Right, Control and calA-treated tubule families after $18 \mathrm{hr}$ culture. (E) Pictographs of family deformation using $\phi$ and $p_{z}$ data from $(F)$. Transparent lines are individual measurements, solid lines are means. (F) Quantitation of $\phi$ and $p_{z}$ for kidneys $+/$ - ureter, and +/- calA for $18 \mathrm{hr}$ (5 measurements per $n=3$ kidneys per condition). Error bars are $\pm S$.D. across all measurements per condition. (C),(F) One-way ANOVA, Tukey's test $\left({ }^{*} p<0.05,{ }^{* *} p<0.01,{ }^{* * *} p<\right.$ $0.001)$. 
If geometric crowding, tip repulsion, and tubule tension are primarily responsible for tip positions, similar outcomes should translate to material systems with similar geometry. We therefore established a biomimetic soft material model of $\sim$ E16 ureteric epithelial families that each share a great-great-grandparent node using 3D printed elastic filaments embedded in a soft silicone elastomer (Fig. 4A, Movie S4). Applying compressive strains in $x y$ that mimic tip crowding by duplication and elongation strains in $z$ were sufficient to create overlapping tip defects and vertical tip transitions, even in a purely abiotic system (Fig. 4B-D, Supplementary Text).

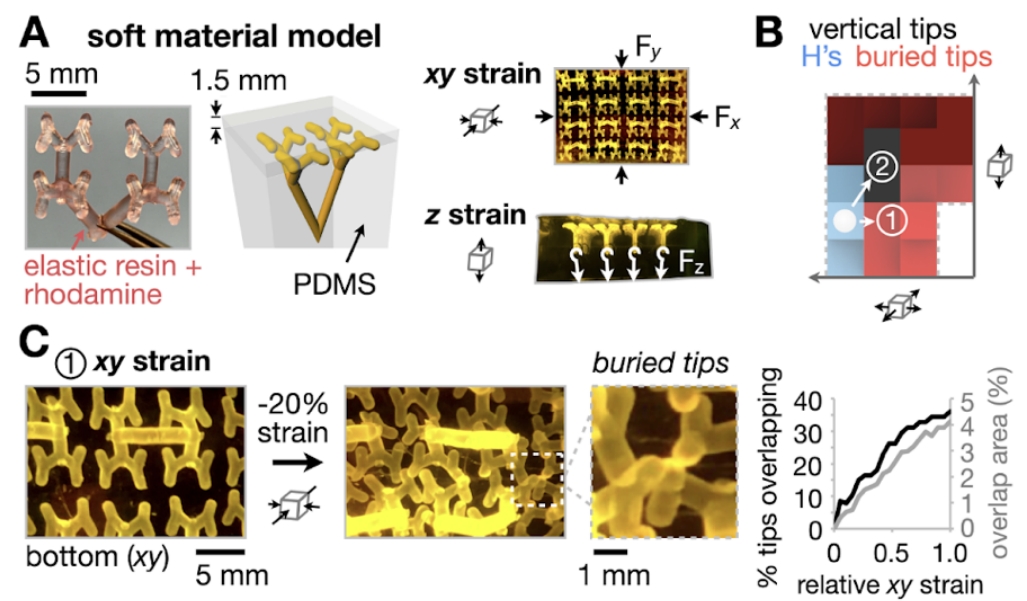

D(2) $z$ strain
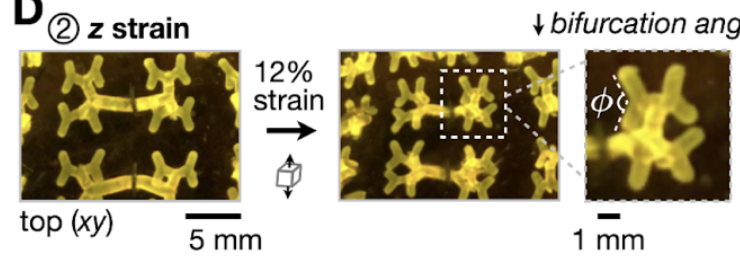

E caps
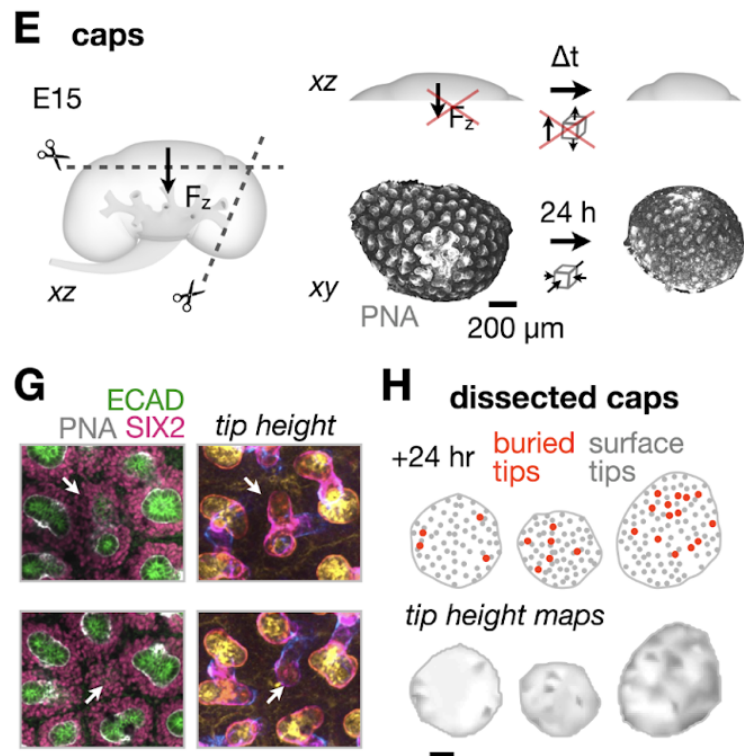

$5 \overline{0} \mu \mathrm{m}$
H dissected caps

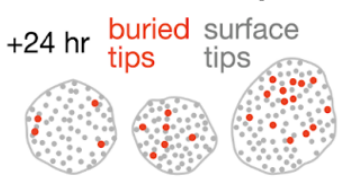

tip height maps
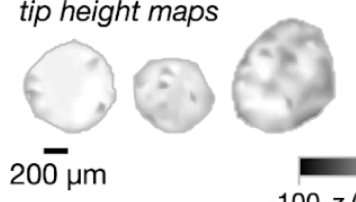

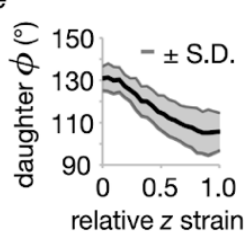

F vertical tips H's buried tips

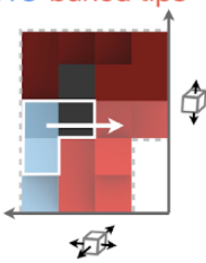

\section{intact controls}

+24 hr patches on
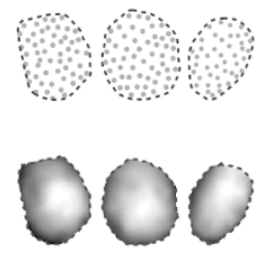

$-100 z(\mu \mathrm{m}) 0$ $+24 \mathrm{hr}$ surface
Fig. 4. Retrograde tubule tension is necessary to avoid buried tips. (A) Left, 3D-printed elastic tubule family. Middle, Rendering of embedding in polydimethylsiloxane (PDMS) silicone. Right, Top and side-view of tubule family array and deformation modes. (B) 2D section of phase space showing expected transitions under strain in $x y$ and $z$. (C) Left, Photographs of tubule positions taken from underside of the model before and after strain is applied in xy. Right, Quantitation of tip overlap metrics. (D) Left, Similar photographs for added strain in $z$. Right, Quantitation of daughter branch bifurcation angle with \pm S.D. envelope. (E) Left and top, cartoon of cap dissection and change in strain parameters over time in culture. Bottom, confocal projections of a cap before and after $24 \mathrm{~h}$ culture. (F) 2D section of phase space showing expected transition for caps in culture. (G) Buried tips shown as left, confocal immunofluorescence image, right, depth-coded projection; one example per image row. (H) Top, pictographs of surface and buried tip locations after $24 \mathrm{~h}$ culture.

Bottom, tip height maps interpolated from $(x, y, z)$ positions of tips. 
We then attempted to force a forbidden phase transition predicted by the models in live explants by removing $\mathrm{F}_{\mathrm{z}}$ from the ureteric tree during the transition to vertical tubule orientation. We dissected 'caps' of cortex from E15 kidneys to sever tubules (and remove force transmission) roughly below the great-grandparent nodes and cultured them for $24 \mathrm{hr}$ (Fig. 4E). These caps compacted in area in the $x y$ plane by $10 \pm 7 \%$ ( $n=6$ caps, Table S1), accentuating planar confinement of tubule families. Our computational and soft material models predict that this would create buried rather than vertical tips near the kidney surface (Fig. 4F). Accordingly, we found that 34 of 290 tips were buried below the surface of dissected caps after $24 \mathrm{hr}$ (across $n=$ 4 caps), whereas none of 161 tips were buried in intact control kidneys (across $n=4$ kidneys) (Fig. 4G,H). Buried tips were visible as 'dimples' in tip height maps for dissected caps after 24 $\mathrm{hr}$, relative to smooth maps for intact controls and caps fixed immediately after cutting (Fig. 4H, Fig. S4). This experiment revealed that retraction forces on tubules are necessary for the ureteric tree to resolve into vertical packing without generating buried tip defects.

Finally, we wondered if our model could predict the impacts of developmental defects on the geometry of the ureteric tree. Genetic mutations and environmental factors including prematurity and nutrient deficiency can affect kidney size and nephron number $(3,22,23)$, branch organization of the ureteric tree (24), and the risk of fetally programmed adult diseases (3). We successfully mapped three published mutants to regressive or forbidden model phases (Fig. S5, Supplementary Text), revealing that the model morphospace may enable insight into abnormal cell and tissue dynamics contributing to an observed tree morphology.

Here we use physics-based simulations, soft material modeling, and kidney explants to reveal a conflict between kidney surface area and exponential duplication of ureteric epithelial tips there. One outcome of this conflict is the emergence of defective packing states. These must be actively avoided through tubule tension toward vertical tip packing. We propose that similar conflicts between increased function and geometric constraints may be at play in other complex organ systems, perhaps amplifying the structural consequences of congenital defects.

\section{References and Notes:}

1. J. M. Smith, Optimization Theory in Evolution. Annu. Rev. Ecol. Syst. 9, 31-56 (1978).

2. B. Mauroy, M. Filoche, E. R. Weibel, B. Sapoval, An optimal bronchial tree may be dangerous. Nature. 427, 633-636 (2004).

3. V. A. Luyckx, J. F. Bertram, B. M. Brenner, C. Fall, W. E. Hoy, S. E. Ozanne, B. E. Vikse, Effect of fetal and child health on kidney development and long-term risk of hypertension and kidney disease. Lancet. 382, 273-283 (2013).

4. F. Costantini, R. Kopan, Patterning a complex organ: branching morphogenesis and nephron segmentation in kidney development. Dev. Cell. 18, 698-712 (2010).

5. T. J. Carroll, J.-S. Park, S. Hayashi, A. Majumdar, A. P. McMahon, Wnt9b plays a central role in the regulation of mesenchymal to epithelial transitions underlying organogenesis of the mammalian urogenital system. Dev. Cell. 9, 283-292 (2005).

6. W. Yu, W. F. Marshall, R. J. Metzger, P. R. Brakeman, L. Morsut, W. Lim, K. E. Mostov, Simple Rules Determine Distinct Patterns of Branching Morphogenesis. Cell Syst. 9, 221-227 (2019).

7. I. M. Smyth, Development of the metanephric kidney. Curr. Top. Dev. Biol. 143, 111-150 (2021). 
8. R. J. Metzger, O. D. Klein, G. R. Martin, M. A. Krasnow, The branching programme of mouse lung development. Nature. 453, 745-750 (2008).

9. A. N. Combes, J. G. Lefevre, S. Wilson, N. A. Hamilton, M. H. Little, Cap mesenchyme cell swarming during kidney development is influenced by attraction, repulsion, and adhesion to the ureteric tip. Dev. Biol. 418, 297-306 (2016).

10. Z. Yao, Stress-induced ordering of two-dimensional packings of elastic spheres. Phys Rev E. 101, 062904 (2020).

11. M. Zu, P. Tan, N. Xu, Forming quasicrystals by monodisperse soft core particles. Nat. Commun. 8, 2089 (2017).

12. W. L. Miller, A. Cacciuto, Two-dimensional packing of soft particles and the soft generalized Thomson problem. Soft Matter. 7, 7552-7559 (2011).

13. A. R. Bausch, M. J. Bowick, A. Cacciuto, A. D. Dinsmore, 4. M. F. Hsu, D. R. Nelson, M. G. Nikolaides, 5. A. Travesset, D. A. Weitz, Grain Boundary Scars and Spherical Crystallography. Science. 299 (2003).

14. E. Kroner, K. H. Anthony, Dislocations and Disclinations in Material Structures: The Basic Topological Concepts. Annu. Rev. Mater. Sci. 5, 43-72 (1975).

15. J. G. Lefevre, K. M. Short, T. O. Lamberton, O. Michos, D. Graf, I. M. Smyth, N. A. Hamilton, Branching morphogenesis in the developing kidney is governed by rules that pattern the ureteric tree. Development. 144, 4377-4385 (2017).

16. K. M. Short, A. N. Combes, J. Lefevre, A. L. Ju, K. M. Georgas, T. Lamberton, O. Cairncross, B. A. Rumballe, A. P. McMahon, N. A. Hamilton, I. M. Smyth, M. H. Little, Global quantification of tissue dynamics in the developing mouse kidney. Dev. Cell. 29, 188-202 (2014).

17. N. O. Lindström, J. A. McMahon, J. Guo, T. Tran, Q. Guo, E. Rutledge, R. K. Parvez, G. Saribekyan, R. E. Schuler, C. Liao, A. D. Kim, A. Abdelhalim, S. W. Ruffins, M. E. Thornton, L. Baskin, B. Grubbs, C. Kesselman, A. P. McMahon, Conserved and Divergent Features of Human and Mouse Kidney Organogenesis. J. Am. Soc. Nephrol. 29, 785-805 (2018).

18. V. Osathanondh, E. L. Potter, DEVELOPMENT OF HUMAN KIDNEY AS SHOWN BY MICRODISSECTION. II. RENAL PELVIS, CALYCES, AND PAPILLAE. Arch. Pathol. 76, 277289 (1963).

19. N. O. Lindström, C.-H. Chang, M. T. Valerius, P. Hohenstein, J. A. Davies, Node retraction during patterning of the urinary collecting duct system. J. Anat. 226, 13-21 (2015).

20. K. S. Stenn, R. Link, G. Moellmann, J. Madri, E. Kuklinska, Dispase, a neutral protease from Bacillus polymyxa, is a powerful fibronectinase and type IV collagenase. J. Invest. Dermatol. 93, 287-290 (1989).

21. A. E. Shyer, A. R. Rodrigues, G. G. Schroeder, E. Kassianidou, S. Kumar, R. M. Harland, Emergent cellular self-organization and mechanosensation initiate follicle pattern in the avian skin. Science. 357, 811-815 (2017). 
22. K. M. Moritz, R. R. Singh, M. E. Probyn, K. M. Denton, Developmental programming of a reduced nephron endowment: more than just a baby's birth weight. Am. J. Physiol. Renal Physiol. 296, F1-9 (2009).

23. S. A. Elmore, S. L. Kavari, M. J. Hoenerhoff, B. Mahler, B. E. Scott, K. Yabe, J. C. Seely, Histology Atlas of the Developing Mouse Urinary System With Emphasis on Prenatal Days E10.5-E18.5. Toxicol. Pathol. 47, 865-886 (2019).

24. M. M. Shah, R. V. Sampogna, H. Sakurai, K. T. Bush, S. K. Nigam, Branching morphogenesis and kidney disease. Development. 131, 1449-1462 (2004).

25. N. Wanek, K. Muneoka, G. Holler-Dinsmore, R. Burton, S. V. Bryant, A staging system for mouse limb development. J. Exp. Zool. 249, 41-49 (1989).

26. S. Bouaziz, S. Martin, T. Liu, L. Kavan, M. Pauly, Projective dynamics: fusing constraint projections for fast simulation. ACM Trans. Graph. 33, 1-11 (2014).

27. M. R. Barnes, Form Finding and Analysis of Tension Structures by Dynamic Relaxation. Int. J. Space Struct. 14, 89-104 (1999).

28. A. N. Combes, K. M. Short, J. Lefevre, N. A. Hamilton, M. H. Little, I. M. Smyth, An integrated pipeline for the multidimensional analysis of branching morphogenesis. Nat. Protoc. 9, 28592879 (2014).

29. L. L. O’Brien, A. N. Combes, K. M. Short, N. O. Lindström, P. H. Whitney, L. A. Cullen-McEwen, A. Ju, A. Abdelhalim, O. Michos, J. F. Bertram, I. M. Smyth, M. H. Little, A. P. McMahon, Wnt11 directs nephron progenitor polarity and motile behavior ultimately determining nephron endowment. Elife. 7 (2018), doi:10.7554/eLife.40392.

30. H. Hama, H. Kurokawa, H. Kawano, R. Ando, T. Shimogori, H. Noda, K. Fukami, A. SakaueSawano, A. Miyawaki, Scale: a chemical approach for fluorescence imaging and reconstruction of transparent mouse brain. Nat. Neurosci. 14, 1481-1488 (2011).

31. H. Morales-Navarrete, H. Nonaka, A. Scholich, F. Segovia-Miranda, W. de Back, K. Meyer, R. L. Bogorad, V. Koteliansky, L. Brusch, Y. Kalaidzidis, F. Jülicher, B. M. Friedrich, M. Zerial, Liquid-crystal organization of liver tissue. Elife. 8 (2019), doi:10.7554/eLife.44860.

32. A. A. Mercurieva, T. M. Birshtein, Liquid-crystalline ordering in two-dimensional systems with discrete symmetry. Makromol. Chem. Theory Simul. 1, 205-214 (1992).

33. P. G. de Gennes, J. Prost, The Physics of Liquid Crystals (Clarendon Press, 1993).

34. B. A. Rumballe, K. M. Georgas, A. N. Combes, A. L. Ju, T. Gilbert, M. H. Little, Nephron formation adopts a novel spatial topology at cessation of nephrogenesis. Dev. Biol. 360, 110122 (2011).

35. J. A. Davies, P. Hohenstein, C.-H. Chang, R. Berry, A self-avoidance mechanism in patterning of the urinary collecting duct tree. BMC Dev. Biol. 14, 35 (2014).

36. Y. Uchiyama, M. Sakaguchi, T. Terabayashi, T. Inenaga, S. Inoue, C. Kobayashi, N. Oshima, H. Kiyonari, N. Nakagata, Y. Sato, K. Sekiguchi, H. Miki, E. Araki, S. Fujimura, S. S. Tanaka, R. Nishinakamura, Kif26b, a kinesin family gene, regulates adhesion of the embryonic kidney mesenchyme. Proc. Natl. Acad. Sci. U. S. A. 107, 9240-9245 (2010). 
37. A. Ihermann-Hella, T. Hirashima, J. Kupari, K. Kurtzeborn, H. Li, H. N. Kwon, C. Cebrian, A. Soofi, A. Dapkunas, I. Miinalainen, G. R. Dressler, M. Matsuda, S. Kuure, Dynamic MAPK/ERK Activity Sustains Nephron Progenitors through Niche Regulation and Primes Precursors for Differentiation. Stem Cell Reports. 11, 912-928 (2018).

38. M. H. Little, Returning to kidney development to deliver synthetic kidneys. Dev. Biol. 474, 22-36 (2021).

39. N. S. R. Maluf, J. J. Gassman, Kidneys of the Killerwhale and Significance of Reniculism. Anat. Rec. 250, 34-44 (1998).

40. L. Chi, U. Saarela, A. Railo, R. Prunskaite-Hyyryläinen, I. Skovorodkin, S. Anthony, K. Katsu, Y. Liu, J. Shan, A. M. Salgueiro, J. A. Belo, J. Davies, Y. Yokouchi, S. J. Vainio, A secreted BMP antagonist, Cer1, fine tunes the spatial organization of the ureteric bud tree during mouse kidney development. PLoS One. 6, e27676 (2011).

41. C. Cebrian, N. Asai, V. D'Agati, F. Costantini, The number of fetal nephron progenitor cells limits ureteric branching and adult nephron endowment. Cell Rep. 7, 127-137 (2014).

42. M. A. Basson, S. Akbulut, J. Watson-Johnson, R. Simon, T. J. Carroll, R. Shakya, I. Gross, G. R. Martin, T. Lufkin, A. P. McMahon, P. D. Wilson, F. D. Costantini, I. J. Mason, J. D. Licht, Sprouty1 is a critical regulator of GDNF/RET-mediated kidney induction. Dev. Cell. 8, 229-239 (2005).

43. M. A. Basson, J. Watson-Johnson, R. Shakya, S. Akbulut, D. Hyink, F. D. Costantini, P. D. Wilson, I. J. Mason, J. D. Licht, Branching morphogenesis of the ureteric epithelium during kidney development is coordinated by the opposing functions of GDNF and Sprouty1. Dev. Biol. 299, 466-477 (2006).

44. I. Gross, D. J. Morrison, D. P. Hyink, K. Georgas, M. A. English, M. Mericskay, S. Hosono, D. Sassoon, P. D. Wilson, M. Little, J. D. Licht, The receptor tyrosine kinase regulator Sprouty1 is a target of the tumor suppressor WT1 and important for kidney development. J. Biol. Chem. 278, 41420-41430 (2003).

\section{Acknowledgements:}

General: We would like to thank Hughes lab members and instructors and students of the 2021 MBL Embryology course for training, discussion and advice. We are grateful to A. Gupta, K. Sasaki, and Y. Sakata for technical assistance with kidney dissections; and to L. Bugaj, A. Raj, and W. Marshall for feedback on the manuscript.

Funding: This work was supported by:

NIH F32 fellowship DK126385 (LSP)

Predoctoral Training Program in Developmental Biology T32HD083185 (JMV)

NIH MIRA R35GM133380 (AJH)

NSF CAREER award 2047271 (AJH)

\section{Author contributions:}

Conceptualization: LSP, AJH

Methodology: LSP, JMV, JL, AJH 


\title{
Software: AJH
}

Formal analysis: LSP, AJH

Investigation: LSP, JMV, JL, AJH

Writing - original draft: LSP, AJH

Writing - review and editing: LSP, JMV, JL, AJH

Visualization: LSP, AJH

Supervision: LSP, AJH

Project administration: AJH

Competing interests: Authors declare that they have no competing interests.

Data and materials availability: All data necessary to evaluate conclusions of this study are presented in the paper and supplementary materials. In-house code and raw data are available from the authors upon request.

\author{
Supplementary Materials \\ Materials and Methods \\ Supplementary Text \\ Figs. S1 to S5 \\ Table S1 \\ Movie S1 to S4 \\ Computational model file
}

